\title{
Real Power Loss Reduction by Hybridization of Tree-Seed Algorithm with Sine-Cosine Algorithm
}

\author{
Kanagasabai Lenin \\ Department of EEE, Prasad V. Potluri Siddhartha Institute of Technology, Kanuru, Vijayawada, Andhra Pradesh, India.
}

\begin{abstract}
How to cite this paper: Kanagasabai Lenin. (2021) Real Power Loss Reduction by Hybridization of Tree-Seed Algorithm with Sine-Cosine Algorithm. Journal of Electrical Power \& Energy Systems, 5(1), 8-23.

DOI: 10.26855/jepes.2021.03.002
\end{abstract}

Received: January 28, 2021

Accepted: February 25, 2021

Published: March 15, 2021

*Corresponding author: Kanagasabai Lenin, Department of EEE, Prasad V. Potluri Siddhartha Institute of Technology, Kanuru, Vijayawada, Andhra Pradesh, India.

Email: gklenin@gmail.com

\begin{abstract}
In this work, real power loss has done through hybridized Tree-seed algorithm. Sine-cosine algorithm which has been combined with Tree-seed algorithm (HTS) is projected to solve the problem. Tree-seed algorithm is based on the relationship between trees and seeds. And Sine Cosine Algorithm is based on the functions of Sine and Cosine; it stimulates the leader variable agent solutions towards the most excellent solution. In this work, seed engendering mechanism has been enhanced through adaptive mode and with reference to the iterations a linearly $(\mathrm{k})$ varying mechanism has been implemented to perk up the exploration and exploitation. With considering voltage stability index proposed hybridized Tree-seed algorithm (HTS) is tested in IEEE 30, bus system. Then, the Proposed hybridized Tree-seed algorithm has been tested in standard IEEE 14, 30, 57, 118, 300 bus test systems without considering the voltage stability index. In first analysis with considering voltage stability index real power loss minimization, voltage deviation minimization, and voltage stability index enhancement has been attained. In the second evaluation without considered voltage stability index, also power loss reduction achieved. Percentage of power loss reduction is $15.80 \%, 20.74 \%$, $26.29 \%$, and $14.55 \%$ with respect to the base value. Power Loss comparison has been done with other standard methods.
\end{abstract}

\section{Keywords}

Optimal Reactive Power, Transmission Loss, Tree-Seed, Sine-Cosine

\section{Introduction}

Minimization of real power loss, voltage stability improvement and deviation of voltage are the main objectives in this work. Many conventional methods like Newton's method, interior point method; successive quadratic programming method [1-6] and evolutionary algorithms like gravitational search, particle swarm optimization, symbiotic organism search algorithm [7-48] are utilized to solve the problem. Conventional methods faced difficulty in handling the constraints. Evolutionary methods have to balance the exploration and exploitation then only optimal solution can be obtained. Many algorithm fail to balance the exploration and exploitation. In this work, Tree-seed algorithm has been combined with sine-cosine algorithm (HTS) to solve the problem. Tree-seed algorithm modeling is based on the tree, seed and during the search a converse association has been preserved between the exploration and exploitation. Sine Cosine Algorithm is based on the functions of Sine and Cosine and it induce prime variable 
agent towards best solution. Tree location has been taken into consideration where seeds are produced and also it will be as best location for the trees. In explore of the optimal values, dispersal of the seeds is very crucial and it is not possible to engender the seeds are not easy in capricious mode. So the number of seeds updating will start from highest and will be gradually reduced to minimum level. Then a novel linear parameter " $K$ " has been employed which has been stimulated from sine cosine algorithm which persuades the solution to jump out from local environment. Hybridization of Tree-seed algorithm with sine-cosine algorithm will improve the exploration and exploitation effectively. Proposed Tree-seed algorithm has been combined with sine-cosine algorithm (HTS) is tested in IEEE 30, bus system with considering voltage stability evaluation index. Then the Proposed Tree-seed algorithm has been combined with sine-cosine algorithm (HTS) has been tested in standard IEEE 14, 30, 57, 118, 300 bus test systems without considering the voltage stability index. Power loss and voltage deviation minimization has been achieved with enhancement of voltage stability index.

\section{Problem Formulation}

Objective function defined as,

Subject to

$$
\text { Minimization } \tilde{F}(\bar{x}, \bar{y})
$$

$$
\begin{gathered}
E(\bar{x}, \bar{y})=0 \\
I(\bar{x}, \bar{y})=0 \\
x=\left[V G_{1}, \ldots, V G_{N g} ; Q C_{1}, \ldots, Q C_{N c} ; T_{1}, \ldots, T_{N_{T}}\right] \\
y=\left[P G_{\text {slack }} ; V L_{1}, \ldots, V L_{N_{\text {Load }}} ; Q G_{1}, \ldots, Q G_{N g} ; S L_{1}, \ldots, S L_{N_{T}}\right]
\end{gathered}
$$

Fitness function $\left(O F_{1}\right)$ is defined to diminish the power loss (MW) is,

$$
O F_{1}=P_{\text {Min }}=\operatorname{Min}\left[\sum_{m}^{N T L} G_{m}\left[V_{i}^{2}+V_{j}^{2}-2 * V_{i} V_{j} \cos \emptyset_{i j}\right]\right]
$$

Fitness function $\left(\mathrm{OF}_{2}\right)$ for minimization of voltage deviation minimization,

$$
O F_{2}=\operatorname{Min}\left[\sum_{i=1}^{N_{L B}}\left|V_{L k}-V_{L k}^{\text {desired }}\right|^{2}+\sum_{i=1}^{N g}\left|Q_{G K}-Q_{K G}^{L i m}\right|^{2}\right]
$$

Fitness function $\left(\mathrm{OF}_{3}\right)$ for voltage stability index value,

$$
\begin{aligned}
& O F_{3}=\operatorname{Min}_{\text {Max }} \\
& L_{\text {Max }}=\operatorname{Max}\left[L_{j}\right] ; j=1 ; N_{L B} \\
& \left\{\begin{array}{c}
L_{j}=1-\sum_{i=1}^{N P V} F_{j i} \frac{V_{i}}{V_{j}} \\
F_{j i}=-\left[Y_{1}\right]^{1}\left[Y_{2}\right] \\
L_{\text {Max }}=\operatorname{Max}\left[1-\left[Y_{1}\right]^{-1}\left[Y_{2}\right] \times \frac{V_{i}}{V_{j}}\right]
\end{array}\right.
\end{aligned}
$$

Equality constraints

$$
\begin{aligned}
& 0=P G_{i}-P D_{i}-V_{i} \sum_{j \in N_{B}} V_{j}\left[G_{i j} \cos \left[\emptyset_{i}-\emptyset_{j}\right]+B_{i j} \sin \left[\emptyset_{i}-\emptyset_{j}\right]\right] \\
& 0=Q G_{i}-Q D_{i}-V_{i} \sum_{j \in N_{B}} V_{j}\left[G_{i j} \sin \left[\emptyset_{i}-\emptyset_{j}\right]+B_{i j} \cos \left[\emptyset_{i}-\emptyset_{j}\right]\right]
\end{aligned}
$$

Inequality constraints

$$
\begin{aligned}
& \mathbf{P}_{\text {gslack }}^{\min } \leq \mathbf{P}_{\text {gslack }} \leq \mathbf{P}_{\text {gslack }}^{\max } \\
& \mathbf{Q}_{\mathrm{gi}}^{\min } \leq \mathbf{Q}_{\mathrm{gi}} \leq \mathbf{Q}_{\mathrm{gi}}^{\max }, \mathbf{i} \in \mathbf{N}_{\mathrm{g}} \\
& \mathrm{VL}_{\mathbf{i}}^{\text {min }} \leq \mathrm{VL}_{\mathbf{i}} \leq \mathrm{VL}_{\mathbf{i}}^{\text {max }}, \mathbf{i} \in \mathbf{N L} \\
& \mathbf{T}_{\mathbf{i}}^{\text {min }} \leq \mathbf{T}_{\mathbf{i}} \leq \mathbf{T}_{\mathbf{i}}^{\max }, \mathbf{i} \in \mathbf{N}_{\mathbf{T}} \\
& \mathrm{Q}_{\mathrm{c}}^{\min } \leq \mathrm{Q}_{\mathrm{c}} \leq \mathrm{Q}_{\mathrm{C}}^{\max }, \mathrm{i} \in \mathrm{N}_{\mathrm{C}}
\end{aligned}
$$




$$
\begin{aligned}
& \left|S L_{i}\right| \leq S_{L_{i}}^{\max }, \mathrm{i} \in \mathrm{N}_{\mathrm{TL}} \\
& \mathrm{VG}_{\mathrm{i}}^{\text {min }} \leq \mathrm{VG}_{\mathrm{i}} \leq \mathrm{VG}_{\mathrm{i}}^{\text {max }}, \mathrm{i} \in \mathrm{N}_{\mathrm{g}}
\end{aligned}
$$

Multi objective fitness (MOF) function is,

$$
\begin{aligned}
& M O F=O F_{1}+x_{i} O F_{2}+y O F_{3}=O F_{1}+\left[\sum_{i=1}^{N L} x_{v}\left[V L_{i}-V L_{i}^{m i n}\right]^{2}+\sum_{i=1}^{N G} x_{g}\left[Q G_{i}-Q G_{i}^{m i n}\right]^{2}\right]+x_{f} O F_{3} \\
& V L_{i}^{\min }=\left\{\begin{array}{l}
V L_{i}^{\max }, V L_{i}>V L_{i}^{\max } \\
V L_{i}^{\min }, V L_{i}<V L_{i}^{\min }
\end{array}\right. \\
& Q G_{i}^{\min }=\left\{\begin{array}{c}
Q G_{i}^{\max }, Q G_{i}>Q G_{i}^{\max } \\
Q G_{i}^{\min }, Q G_{i}<Q G_{i}^{\min }
\end{array}\right.
\end{aligned}
$$

\section{Hybridization of Tree-Seed Algorithm with Sine-Cosine Algorithm}

In this work, Tree-seed algorithm has been combined with sine-cosine algorithm (HTC) for power loss reduction. Tree-seed algorithm is based on the association between trees and seeds. Many problems have been solved by the Tree-seed algorithm effectively but it lacks in the global searching and also there is limitation in the exploitation also. In order to overcome these defects, Tree-seed algorithm has been combined with sine-cosine algorithm for solving the problem. Sine Cosine Algorithm is based on the functions of Sine and Cosine; it excites principal variable agent solutions and it sways externally or inner mode towards the most excellent solution. In this work, seed engendering mechanism has been improved in adaptive mode and with respect to the iterations a linearly (k) varying mechanism has been implemented.

Tree-seed algorithm modeling is based on the tree, seed and during searching an inverse correlation has been maintained between the exploration and exploitation.

$$
\begin{aligned}
& f(\vec{G}) \leq f(\vec{H}), \forall \vec{H} \in P \\
& f(\vec{G}) \geq f(\vec{H}), \forall \vec{H} \in P
\end{aligned}
$$

Where " $f$ " the objective function and "P" is the solutions which are suitable.

Tree position has been taken into consideration where seeds are engendered and also it will be as most excellent location for the population of the trees [44-47]. Then the updating equation can be mathematically written as,

$$
\begin{aligned}
& D m_{i, j}=\operatorname{Trd}_{i, j}+\alpha_{i, j} \times\left(C_{j}-\operatorname{Tr}_{e, j}\right) \\
& D m_{i, j}=\operatorname{Trd}_{i, j}+\alpha_{i, j} \times\left(\operatorname{Trd}_{i, j}-\operatorname{Tr}_{e, j}\right)
\end{aligned}
$$

Where $D m_{i, j}$ is the ith seeds jth dimension, $\operatorname{Trd}_{i, j}$ is the ith tree jth dimension, $C_{j}$ is the most excellent jth tree location or position obtained, $\operatorname{Tr}_{e, j}$ is the rth tree in jth dimension arbitrarily chosen from the population, $\alpha_{i, j}$ is the scaling factor from $[-1,1]$.

In the algorithm, preliminary tree locations or positions will be the beginning of the exploration procedure and it engendered by,

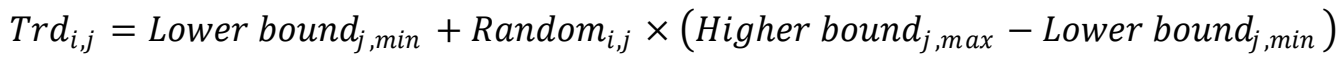

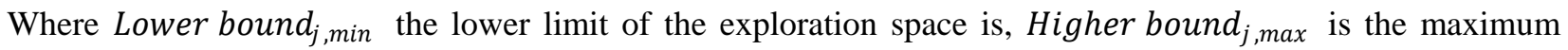
limit of the exploration space, Random $_{i, j}$ is the number arbitrarily produced in range of $[0,1]$.

From the population, the best solution has been selected to comprehend the minimization by,

$$
C=\operatorname{minimum}\left(f\left(\overrightarrow{\operatorname{Tr}_{i}}\right)\right)
$$

Then, the maximum number of function evaluation is given by,

$$
\text { Maximum function evaluation }=\text { Dimensionality } \times 10000
$$
a. Start;
b. Set the preliminary values and end criterion
c. In the exploration space engender " $N$ " trees arbitrarily 


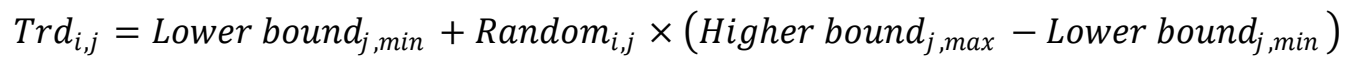

d. Then, the most excellent solution obtained so far will be saved

$$
C=\operatorname{minimum}\left(f\left(\overrightarrow{T r}_{i}\right)\right)
$$

e. For every tree number of seeds to be produced should be determined

f. Is random $<S T$ ?

g. If Yes update the dimension by,

$$
D m_{i, j}=\operatorname{Trd}_{i, j}+\alpha_{i, j} \times\left(C_{j}-\operatorname{Tr}_{e, j}\right)
$$

h. If No update the dimension by

$$
D m_{i, j}=\operatorname{Trd}_{i, j}+\alpha_{i, j} \times\left(\operatorname{Tr} d_{i, j}-\operatorname{Tr}_{e, j}\right)
$$

i. Is passing through each seed of every tree?

$\mathrm{j}$. If Yes then choose the most excellent seed and compare with the tree

k. If No the go to step " $\mathrm{b}$ "

1. Is the value of the objective function better than preceding one?

$\mathrm{m}$. If yes then the position of the seeds are the alternate for the trees

n. If No form the population choose the most excellent solution by,

$$
C=\operatorname{minimum}\left(f\left(\overrightarrow{T r_{i}}\right)\right)
$$

o. Is the new most excellent solution better than preceding one?

p. If Yes then new most excellent solution will substitute the preceding solution

q. If No then the preceding solution is the most excellent one

r. Is the end condition satisfied?

s. If Yes then report the most excellent solution

t. If No then go to step "e"

u. End

Sine Cosine Algorithm based on Sine and Cosine functions ; it produces preliminary arbitrary agent solutions and it swing outwardly or inward towards the most excellent solution by using numerical model which based on sine and cosine functions [48].

$$
\begin{aligned}
& \vec{X}_{i}^{t+1}=\vec{X}_{i}^{t}+\text { random }_{1} \times \sin \left(\text { random }_{2}\right) \times \mid \text { random }_{3} \times I_{i}^{t}-\vec{X}_{i}^{t} \mid \\
& \vec{X}_{i}^{t+1}=\vec{X}_{i}^{t}+\text { random }_{1} \times \cos \left(\text { random }_{2}\right) \times \mid \text { random }_{3} \times I_{i}^{t}-\vec{X}_{i}^{t} \mid \\
& \vec{X}_{i}^{t+1}=\left\{\begin{array}{l}
\vec{X}_{i}^{t}+\text { random }_{1} \times \sin \left(\text { random }_{2}\right) \times \mid \text { random }_{3} \times I_{i}^{t}-\vec{X}_{i}^{t} \mid \text { random }_{4}<0.5 \\
\vec{X}_{i}^{t}+\operatorname{random}_{1} \times \cos \left(\text { random }_{2}\right) \times \mid \text { random }_{3} \times I_{i}^{t}-\vec{X}_{i}^{t} \mid \text { random }_{4} \geq 0.5
\end{array}\right.
\end{aligned}
$$

a. Start

b. Initialize the search agents

c. Is $t<$ maximum number of iterations?

d. If Yes agents are evaluated based on the fitness values

e. Then update the most excellent solution

f. Modernize the parameters

g. Then the position of the search agents are updated by

$$
\begin{aligned}
& \vec{X}_{i}^{t+1}=\vec{X}_{i}^{t}+\text { random }_{1} \times \sin \left(\text { random }_{2}\right) \times \mid \text { random }_{3} \times I_{i}^{t}-\vec{X}_{i}^{t} \mid \\
& \vec{X}_{i}^{t+1}=\vec{X}_{i}^{t}+\text { random }_{1} \times \cos \left(\text { random }_{2}\right) \times \mid \text { random }_{3} \times I_{i}^{t}-\vec{X}_{i}^{t} \mid
\end{aligned}
$$

h. If No then report the most excellent solution obtained so far

i. End

In search of the optimal values of spreading of seeds is very essential and it is not possible to stimulate the seeds are not easy in arbitrary mode. So the number of seeds updating will start from maximum and will be steadily reduced. 
Then the maximum function evaluation written as

$$
\begin{gathered}
\text { Ratio of maximum function evaluation }=\frac{\text { function evaluation }}{\text { maximum function evaluation }} \\
\text { GTheatv }=0.50 \times \text { Ratio function evaluation } \times \pi \\
\text { Number of seeds }=\text { Lower bound }+\mid(\text { Higher bound }- \text { Lower bound }) \times \operatorname{Cos}(\text { GTheatv }) \mid+1.0
\end{gathered}
$$

By changing the number of seeds, the global exploration capability is greatly improved.

Then a new linear parameter " $K$ " has been implemented and it inspired from sine cosine algorithm which induces the solution to jump out from local environment. At first in the process, exploration has been improved then gradually exploitation will be enhanced.

$$
k=2.0 \times(1-\text { Ratio function evaluation })
$$

Then in the Hybridization Tree-seed algorithm with sine-cosine algorithm, the location or the position of the seed is obtained from the tree is very key for the exploration procedure. Then the exploration equation is given by,

$$
\begin{aligned}
& D m_{i, j}=\text { Random } \times \operatorname{Tr}_{i, j}+(1-\text { Random }) \times C_{j}, \text { Random } \leq 0.50 S T \\
& D m_{i, j}=\operatorname{Tr}_{i, j}+k\left(C_{j}-\text { Random } \operatorname{tr}_{i, j} \times \operatorname{Tr}_{i, j}\right) \times\left(\operatorname{Sin}\left(\pi \times \operatorname{acos}\left(\operatorname{Random}_{\mathrm{t}} \boldsymbol{R}_{i, j}\right)\right)\right), 0.50 S T \leq \operatorname{random} \leq S T
\end{aligned}
$$

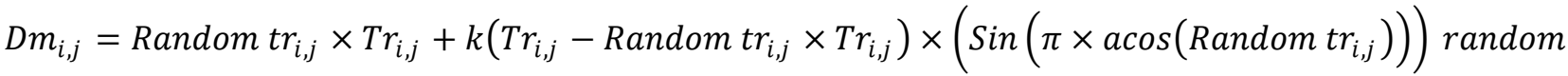

$$
\begin{aligned}
& \geq S T
\end{aligned}
$$

a. Start

b. Number of population is set by,

Number of seeds $=$ Lower bound $+\mid($ Higher bound - Lower bound $) \times \operatorname{Cos}($ GTheatv $) \mid+1.0$

c. Then "K" expansion coefficient is set by,

$$
k=2.0 \times(1-\text { Ratio function evaluation })
$$

d. Value of the parameter ST has been set

e. Problem dimensionality defined

f. End criterion of the process is defined

g. Tree locations or positions engendered arbitrarily by,

$$
\operatorname{Trd}_{i, j}=\text { Lower bound }_{j, \text { min }}+\text { Random }_{i, j} \times\left(\text { Higher bound }_{j, \text { max }}-\text { Lower bound }_{j, \text { min }}\right)
$$

h. With respect to the objective function of the problem location or position of the Tree evaluated

i. Most excellent solution will be chosen

$$
C=\operatorname{minimum}\left(f\left(\overrightarrow{\operatorname{Tr}_{i}}\right)\right)
$$

j. $\quad$ Explore with seeds of the Tree

k. For all Trees

l. For every Tree how many seeds to be produced will be defined

m. For all Seeds

n. For all Dimensions

o. If random $<0.50 \times S T$

p. Modernize the seeds by

$$
D m_{i, j}=\text { Random } \times \operatorname{Tr}_{i, j}+(1-\text { Random }) \times C_{j}, \text { Random } \leq 0.50 S T
$$

q. $\quad$ Else if $(0.50 \times S T \leq$ random $<S T)$

r. Then Modernize the seeds by

$D m_{i, j}=\operatorname{Tr}_{i, j}+k\left(C_{j}-\right.$ Random $\left.t r_{i, j} \times \operatorname{Tr}_{i, j}\right) \times\left(\operatorname{Sin}\left(\pi \times \operatorname{acos}\left(\operatorname{Random}_{\mathrm{tr}} r_{i, j}\right)\right)\right), 0.50 S T \leq \operatorname{random} \leq S T$ 
s. Or else Modernize the seeds by

t. $D m_{i, j}=$

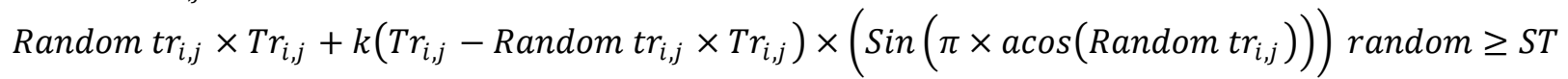

u. $\quad$ End if

v. End for

w. Most excellent seed chosen and it compared with Tree

$\mathrm{x}$. If the objective value of the seed is better then it will substitute the Tree

y. End for

z. Form the population choose the most excellent population by,

$$
\text { Maximum function evaluation }=\text { Dimensionality } \times 10000
$$

aa. When the new most excellent solution is better than preceding solution then it will be replaced

bb. If the end criterion met then stop the process Or else go to step " $\mathrm{j}$ ”

cc. Output the most excellent solution

dd. End

\section{Simulation Results}

Projected Hybridization of Tree-seed algorithm with sine-cosine algorithm (HTS) has been tested in standard IEEE 30 bus system [49]. Active and reactive power consumption is 2.834 and 1.262 per unit on 100 MVA base. Table 1 and Table 2 show the variable parameters. Then comparisons of values are given in Tables 3 to 6 . Power loss comparison has been achieved, voltage stability enhancement with voltage deviation minimization attained.

Table 1. Control variables

\begin{tabular}{cccc}
\hline System [49] & Variables & Minimum (PU) & Maximum (PU) \\
\hline \multirow{3}{*}{ IEEE 30 Bus } & Generator Voltage & 0.95 & 1.1 \\
& Transformer Tap & 0.9 & 1.1 \\
& VAR Source & 0 & 5 (MVAR) \\
\hline
\end{tabular}

Table 2. System parameters

\begin{tabular}{ccc}
\hline Description [49] & IEEE 30 bus \\
\hline NB- number of buses & 30 \\
NG- number of generators & 6 \\
NT- number of transformers & 4 \\
NQ- number of shunt & 9 \\
NE- number of branches & 41 \\
PLoss (base case) MW & 5.66 \\
Base care for VD (PU) & 0.58217 \\
\hline
\end{tabular}

Table 3. Comparison of real power loss

\begin{tabular}{|c|c|c|c|c|}
\hline & $\mathrm{DE}[50]$ & GSA[51] & APOPSO [52] & HTS \\
\hline Gen.Volt1 & 1.1 & 1.071 & 1.100 & 1.093 \\
\hline Gen.Volt2 & 1.09 & 1.022 & 1.084 & 1.041 \\
\hline Gen.Volt5 & 1.07 & 1.040 & 1.056 & 1.020 \\
\hline Gen.Volt8 & 1.07 & 1.051 & 1.076 & 1.032 \\
\hline Gen.Volt11 & 1.1 & 0.977 & 1.091 & 1.090 \\
\hline Gen.Volt13 & 5 & 0.968 & 1.100 & 0.981 \\
\hline
\end{tabular}




\begin{tabular}{|c|c|c|c|c|}
\hline Capacitor Reactive Power 10 & 5 & 1.653 & 5.000 & 4.980 \\
\hline Capacitor Reactive Power 12 & 5 & 4.3722 & 5.000 & 5.001 \\
\hline Capacitor Reactive Power 15 & 5 & 0.1199 & 4.879 & 4.792 \\
\hline Capacitor Reactive Power 17 & 5 & 2.0876 & 4.976 & 4.980 \\
\hline Capacitor Reactive Power 20 & 4.41 & 0.357 & 3.821 & 3.710 \\
\hline Capacitor Reactive Power 21 & 5 & 0.2602 & 4.541 & 4.659 \\
\hline Capacitor Reactive Power 23 & 2.8004 & 0.0000 & 2.354 & 2.407 \\
\hline Capacitor Reactive Power 24 & 5 & 1.3839 & 4.654 & 4.509 \\
\hline Capacitor Reactive Power 29 & 2.5979 & 0.0000 & 2.175 & 2.159 \\
\hline $\mathrm{T} 11(6-9)$ & 1.04 & 1.0985 & 1.029 & 1.009 \\
\hline $\mathrm{T} 12(6-10)$ & 0.9097 & 0.9824 & 0.911 & 0.901 \\
\hline $\mathrm{T} 15$ (4-12) & 0.98 & 1.095 & 0.952 & 0.922 \\
\hline T36 (28-27) & 0.9689 & 1.0593 & 0.958 & 0.939 \\
\hline PLoss (MW) & 4.555 & 4.5143 & 4.398 & 4.220 \\
\hline VD (PU) & 1.9589 & 0.87522 & 1.047 & 1.028 \\
\hline \multirow{3}{*}{ L-index (PU) } & 0.5513 & 0.14109 & 0.1267 & 0.1224 \\
\hline & \multicolumn{4}{|c|}{ Table 4. Comparison of different algorithms } \\
\hline & $\mathrm{DE}[50]$ & GSA [51] & APOPSO [52] & HTS \\
\hline Gen.Volt1 & 1.01 & 0.983 & 1.011 & 1.019 \\
\hline Gen.Volt2 & 0.99 & 1.044 & 1.001 & 1.016 \\
\hline Gen.Volt5 & 1.02 & 1.020 & 1.014 & 1.020 \\
\hline Gen.Volt8 & 1.02 & 0.999 & 1.009 & 1.021 \\
\hline Gen.Volt11 & 1.01 & 1.077 & 0.954 & 0.943 \\
\hline Gen.Volt13 & 1.03 & 1.044 & 1.000 & 1.001 \\
\hline Capacitor Reactive Power 10 & 4.94 & 0 & 4.102 & 4.102 \\
\hline Capacitor Reactive Power 12 & 1.0885 & 0.4735 & 2.124 & 2.119 \\
\hline Capacitor Reactive Power 15 & 4.9985 & 5 & 4.512 & 4.492 \\
\hline Capacitor Reactive Power 17 & 0.2393 & 0 & 0.000 & 0.000 \\
\hline Capacitor Reactive Power 20 & 4.99 & 5 & 5.000 & 5.000 \\
\hline Capacitor Reactive Power 21 & 4.90 & 0 & 5.000 & 5.000 \\
\hline Capacitor Reactive Power 23 & 4.9863 & 4.9998 & 5.000 & 5.000 \\
\hline Capacitor Reactive Power 24 & 4.9663 & 5 & 5.000 & 5.000 \\
\hline Capacitor Reactive Power 29 & 2.2325 & 5 & 4.120 & 4.131 \\
\hline $\mathrm{T} 11(6-9)$ & 1.02 & 0.9 & 0.998 & 0.991 \\
\hline T12 (6-10) & 0.9038 & 1.1 & 0.822 & 0.813 \\
\hline $\mathrm{T} 15(4-12)$ & 1.01 & 1.051 & 0.954 & 0.939 \\
\hline Т36 (28-27) & 0.9635 & 0.9619 & 0.958 & 0.942 \\
\hline PLoss (MW) & 6.4755 & 6.9117 & 5.698 & 5.398 \\
\hline VD (PU) & 0.0911 & 0.0676 & 0.087 & 0.069 \\
\hline L-index (PU) & 0.14352 & 0.1349 & 0.1377 & 0.1335 \\
\hline
\end{tabular}


Table 5. Comparison of values

\begin{tabular}{|c|c|c|c|c|}
\hline & \multicolumn{4}{|c|}{ Methods } \\
\hline & $\mathrm{DE}[50]$ & GSA [51] & APOPSO [52] & HTS \\
\hline Gen.Volt1 & 1.09 & 1.1 & 1.043 & 1.030 \\
\hline Gen.Volt2 & 1.09 & 1.1 & 1.061 & 1.049 \\
\hline Gen.Volt5 & 1.09 & 1.1 & 1.061 & 1.048 \\
\hline Gen.Volt8 & 1.04 & 1.1 & 1.057 & 1.039 \\
\hline Gen.Volt11 & 1.09 & 1.1 & 1.048 & 1.040 \\
\hline Gen.Volt13 & 0.95 & 1.1 & 1.091 & 1.069 \\
\hline Capacitor Reactive Power 10 & 0.69 & 5 & 0.040 & 0.041 \\
\hline Capacitor Reactive Power 12 & 4.7163 & 5 & 0.039 & 0.040 \\
\hline Capacitor Reactive Power 15 & 4.4931 & 5 & 0.038 & 0.042 \\
\hline Capacitor Reactive Power 17 & 4.51 & 5 & 0.040 & 0.041 \\
\hline Capacitor Reactive Power 20 & 4.48 & 5 & 0.037 & 0.037 \\
\hline Capacitor Reactive Power 21 & 4.60 & 5 & 0.009 & 0.019 \\
\hline Capacitor Reactive Power 23 & 3.8806 & 5 & 0.019 & 0.016 \\
\hline Capacitor Reactive Power 24 & 3.8806 & 5 & 0.011 & 0.020 \\
\hline Capacitor Reactive Power 29 & 3.2541 & 5 & 0.001 & 0.007 \\
\hline T11 (6-9) & 0.90 & 0.9 & 0.919 & 0.909 \\
\hline T12 (6-10) & 0.9029 & 0.9 & 0.924 & 0.912 \\
\hline T15 (4-12) & 0.90 & 0.9 & 0.938 & 0.921 \\
\hline T36 (28-27) & 0.936 & 1.0195 & 0.924 & 0.922 \\
\hline PLoss (MW) & 7.0733 & 4.9752 & 4.478 & 4.219 \\
\hline VD (PU) & 1.419 & 0.21579 & 1.8579 & 1.8199 \\
\hline L-index (PU) & 0.1246 & 0.13684 & 0.1227 & 0.1192 \\
\hline
\end{tabular}

Table 6. Comparison of loss

\begin{tabular}{ccc}
\hline & Methods & HTS \\
\hline Gen.Volt1 & APOPSO [52] & 1.014 \\
Gen.Volt2 & 1.020 & 1.021 \\
Gen.Volt5 & 1.033 & 1.000 \\
Gen.Volt8 & 1.000 & 1.000 \\
Gen.Volt11 & 1.004 & 1.019 \\
Gen.Volt13 & 1.032 & 1.022 \\
Capacitor Reactive Power 10 & 1.028 & 0.039 \\
Capacitor Reactive Power 12 & 0.051 & 0.002 \\
Capacitor Reactive Power 15 & 0.002 & 0.039 \\
Capacitor Reactive Power 17 & 0.044 & 0.003 \\
Capacitor Reactive Power 20 & 0.009 & 0.027 \\
Capacitor Reactive Power 21 & 0.048 & 0.041 \\
\hline
\end{tabular}


Capacitor Reactive Power 23

Capacitor Reactive Power 24

Capacitor Reactive Power 29

$$
\begin{gathered}
\text { T11 (6-9) } \\
\text { T12 (6-10) } \\
\text { T15 (4-12) } \\
\text { T36 (28-27) } \\
\text { PLoss (MW) } \\
\text { VD (PU) } \\
\text { L-index (PU) }
\end{gathered}
$$

\begin{tabular}{|c|c|c|c|c|c|c|}
\hline Control variables & Base case & MPSO [56] & PSO [55] & EP [54] & SARGA [54] & HTS \\
\hline Gen.volt-1 & 1.060 & 1.100 & 1.100 & $\mathrm{NR}^{*}$ & NR* & 1.021 \\
\hline Gen.volt-2 & 1.045 & 1.085 & 1.086 & 1.029 & 1.060 & 1.019 \\
\hline Gen.volt-3 & 1.010 & 1.055 & 1.056 & 1.016 & 1.036 & 1.029 \\
\hline Gen.volt-6 & 1.070 & 1.069 & 1.067 & 1.097 & 1.099 & 1.023 \\
\hline Gen.volt -8 & 1.090 & 1.074 & 1.060 & 1.053 & 1.078 & 1.021 \\
\hline Tt 8 & 0.978 & 1.018 & 1.019 & 1.04 & 0.95 & 0.903 \\
\hline Tt 9 & 0.969 & 0.975 & 0.988 & 0.94 & 0.95 & 0.909 \\
\hline Tt 10 & 0.932 & 1.024 & 1.008 & 1.03 & 0.96 & 0.919 \\
\hline capacitor reactive power-9 & 0.19 & 14.64 & 0.185 & 0.18 & 0.06 & 0.142 \\
\hline$P G$ & 272.39 & 271.32 & 271.32 & NR* & NR* & 271.31 \\
\hline$Q G$ (Mvar) & 82.44 & 75.79 & 76.79 & $\mathrm{NR}^{*}$ & $\mathrm{NR}^{*}$ & 74.40 \\
\hline Reduction in PLoss (\%) & 0 & 9.2 & 9.1 & 1.5 & 2.5 & 15.80 \\
\hline Total PLoss (Mw) & 13.550 & 12.293 & 12.315 & 13.346 & 13.216 & 11.409 \\
\hline
\end{tabular}

$\begin{array}{ll}0.033 & 0.030 \\ 0.050 & 0.038 \\ 0.015 & 0.019 \\ 1.042 & 1.037 \\ 0.909 & 0.919 \\ 1.023 & 1.015 \\ 0.958 & 0.936 \\ 4.842 & 4.729 \\ 1.009 & 1.004 \\ 0.1192 & 0.1197\end{array}$

Then in standard IEEE 14 bus system, the validity of the proposed Hybridization of Tree-seed algorithm with sine-cosine algorithm (HTS) has been evaluated without considering voltage stability index. Table 7 shows the comparison results. Power loss reduced and Percentage of power loss increased by $15.80 \%$

Table 7. Simulation results of IEEE-14 system

NR* - Not reported.

Then, the proposed Hybridization of Tree-seed algorithm with sine-cosine algorithm (HTS) has been tested without considering voltage stability index, in IEEE 30 Bus system. Comparison results are presented in Table 8. Power loss reduction percentage value is $20.74 \%$.

Table 8. Simulation results of IEEE-30 system

\begin{tabular}{ccccccc}
\hline Control variables & Base case & MPSO [56] & PSO [55] & EP [54] & SARGA [54] & HTS \\
\hline Gen.Volt-1 & 1.060 & 1.101 & 1.100 & $\mathrm{Nr}^{*}$ & $\mathrm{Nr}^{*}$ & 1.019 \\
Gen.Volt-2 & 1.045 & 1.086 & 1.072 & 1.097 & 1.094 & 1.020 \\
Gen.Volt-5 & 1.010 & 1.047 & 1.038 & 1.049 & 1.053 & 1.039 \\
Gen.Volt-8 & 1.010 & 1.057 & 1.048 & 1.033 & 1.059 & 1.028 \\
Gen.Volt-12 & 1.082 & 1.048 & 1.058 & 1.092 & 1.099 & 1.049 \\
Gen.Volt-13 & 1.071 & 1.068 & 1.080 & 1.091 & 1.099 & 1.043 \\
\hline
\end{tabular}




\begin{tabular}{|c|c|c|c|c|c|c|}
\hline Tt11 & 0.978 & 0.983 & 0.987 & 1.01 & 0.99 & 0.921 \\
\hline $\mathrm{Tt} 12$ & 0.969 & 1.023 & 1.015 & 1.03 & 1.03 & 0.920 \\
\hline Tt15 & 0.932 & 1.020 & 1.020 & 1.07 & 0.98 & 0.911 \\
\hline $\mathrm{Tt} 36$ & 0.968 & 0.988 & 1.012 & 0.99 & 0.96 & 0.910 \\
\hline Capacitor Reactive Power10 & 0.19 & 0.077 & 0.077 & 0.19 & 0.19 & 0.094 \\
\hline Capacitor Reactive Power24 & 0.043 & 0.119 & 0.128 & 0.04 & 0.04 & 0.113 \\
\hline$P G(\mathrm{MW})$ & 300.9 & 299.54 & 299.54 & $\mathrm{NR}^{*}$ & $\mathrm{NR}^{*}$ & 298.41 \\
\hline$Q G$ (Mvar) & 133.9 & 130.83 & 130.94 & $\mathrm{NR}^{*}$ & $\mathrm{NR}^{*}$ & 130.32 \\
\hline Reduction in PLoss (\%) & 0 & 8.4 & 7.4 & 6.6 & 8.3 & 20.74 \\
\hline Total PLoss (Mw) & 17.55 & 16.07 & 16.25 & 16.38 & 16.09 & 13.91 \\
\hline
\end{tabular}

NR* - Not reported.

Then the proposed Hybridization of Tree-seed algorithm with sine-cosine algorithm (HTS) has been tested without considering voltage stability index in IEEE 57 Bus system. Table 9 shows the comparison results and with reference to base case value percentage of loss reduction is $26.29 \%$.

Table 9. Simulation results of IEEE-57 system

\begin{tabular}{|c|c|c|c|c|c|c|}
\hline Control variables & Base case & MPSO [56] & PSO [55] & CGA [53] & AGA [53] & HTS \\
\hline Gen.volt 1 & 1.040 & 1.093 & 1.083 & 0.968 & 1.027 & 1.019 \\
\hline Gen.volt 2 & 1.010 & 1.086 & 1.071 & 1.049 & 1.011 & 1.020 \\
\hline Gen.volt 3 & 0.985 & 1.056 & 1.055 & 1.056 & 1.033 & 1.021 \\
\hline Gen.volt 6 & 0.980 & 1.038 & 1.036 & 0.987 & 1.001 & 1.020 \\
\hline Gen.volt 8 & 1.005 & 1.066 & 1.059 & 1.022 & 1.051 & 1.023 \\
\hline Gen.volt 9 & 0.980 & 1.054 & 1.048 & 0.991 & 1.051 & 1.019 \\
\hline Gen.volt 12 & 1.015 & 1.054 & 1.046 & 1.004 & 1.057 & 1.039 \\
\hline Tt 19 & 0.970 & 0.975 & 0.987 & 0.920 & 1.030 & 0.951 \\
\hline Tt 20 & 0.978 & 0.982 & 0.983 & 0.920 & 1.020 & 0.932 \\
\hline Tt 31 & 1.043 & 0.975 & 0.981 & 0.970 & 1.060 & 0.929 \\
\hline Tt 35 & 1.000 & 1.025 & 1.003 & $\mathrm{NR}^{*}$ & NR* & 1.028 \\
\hline Tt 36 & 1.000 & 1.002 & 0.985 & $\mathrm{NR}^{*}$ & NR* & 1.006 \\
\hline Tt 37 & 1.043 & 1.007 & 1.009 & 0.900 & 0.990 & 1.005 \\
\hline Tt 41 & 0.967 & 0.994 & 1.007 & 0.910 & 1.100 & 0.993 \\
\hline Tt 46 & 0.975 & 1.013 & 1.018 & 1.100 & 0.980 & 1.016 \\
\hline Tt 54 & 0.955 & 0.988 & 0.986 & 0.940 & 1.010 & 0.977 \\
\hline Tt 58 & 0.955 & 0.979 & 0.992 & 0.950 & 1.080 & 0.969 \\
\hline Tt 59 & 0.900 & 0.983 & 0.990 & 1.030 & 0.940 & 0.952 \\
\hline Tt 65 & 0.930 & 1.015 & 0.997 & 1.090 & 0.950 & 1.004 \\
\hline Tt 66 & 0.895 & 0.975 & 0.984 & 0.900 & 1.050 & 0.955 \\
\hline Tt 71 & 0.958 & 1.020 & 0.990 & 0.900 & 0.950 & 1.004 \\
\hline Tt 73 & 0.958 & 1.001 & 0.988 & 1.000 & 1.010 & 1.009 \\
\hline Tt 76 & 0.980 & 0.979 & 0.980 & 0.960 & 0.940 & 0.953 \\
\hline Tt 80 & 0.940 & 1.002 & 1.017 & 1.000 & 1.000 & 1.001 \\
\hline
\end{tabular}




\begin{tabular}{cccccccc}
\hline capacitor reactive power 18 & 0.1 & 0.179 & 0.131 & 0.084 & 0.016 & 0.172 \\
capacitor reactive power 25 & 0.059 & 0.176 & 0.144 & 0.008 & 0.015 & 0.159 \\
capacitor reactive power 53 & 0.063 & 0.141 & 0.162 & 0.053 & 0.038 & 0.144 \\
$P G$ (MW) & 1278.6 & 1274.4 & 1274.8 & 1276 & 1275 & 1267.39 \\
$Q G$ (Mvar) & 321.08 & 272.27 & 276.58 & 309.1 & 304.4 & 271.14 \\
Reduction in PLoss (\%) & 0 & 15.4 & 14.1 & 9.2 & 11.6 & 26.29 \\
Total PLoss (Mw) & 27.8 & 23.51 & 23.86 & 25.24 & 24.56 & 20.49 \\
\hline
\end{tabular}

NR* - Not reported.

Then the proposed Hybridization of Tree-seed algorithm with sine-cosine algorithm (HTS) has been tested without considering voltage stability index in IEEE 118 Bus system. Comparison results are presented in Table 10. Power loss reduction percentage is $14.55 \%$.

Table 10. Simulation results of IEEE-118 system

\begin{tabular}{|c|c|c|c|c|c|c|}
\hline Control variables & Base case & MPSO [56] & PSO [55] & IPSO [55] & CLPSO [53] & HTS \\
\hline Gen.volt 1 & 0.955 & 1.021 & 1.019 & 1.085 & 1.033 & 1.013 \\
\hline Gen.volt 4 & 0.998 & 1.044 & 1.038 & 1.042 & 1.055 & 1.042 \\
\hline Gen.volt 6 & 0.990 & 1.044 & 1.044 & 1.080 & 0.975 & 1.030 \\
\hline Gen.volt 8 & 1.015 & 1.063 & 1.039 & 0.968 & 0.966 & 1.004 \\
\hline Gen.volt 10 & 1.050 & 1.084 & 1.040 & 1.075 & 0.981 & 1.029 \\
\hline Gen.volt 12 & 0.990 & 1.032 & 1.029 & 1.022 & 1.009 & 1.028 \\
\hline Gen.volt 15 & 0.970 & 1.024 & 1.020 & 1.078 & 0.978 & 1.039 \\
\hline Gen.volt 18 & 0.973 & 1.042 & 1.016 & 1.049 & 1.079 & 1.033 \\
\hline Gen.volt 19 & 0.962 & 1.031 & 1.015 & 1.077 & 1.080 & 1.030 \\
\hline Gen.volt 24 & 0.992 & 1.058 & 1.033 & 1.082 & 1.028 & 1.029 \\
\hline Gen.volt 25 & 1.050 & 1.064 & 1.059 & 0.956 & 1.030 & 1.031 \\
\hline Gen.volt 26 & 1.015 & 1.033 & 1.049 & 1.080 & 0.987 & 1.037 \\
\hline Gen.volt 27 & 0.968 & 1.020 & 1.021 & 1.087 & 1.015 & 0.901 \\
\hline Gen.volt31 & 0.967 & 1.023 & 1.012 & 0.960 & 0.961 & 0.903 \\
\hline Gen.volt 32 & 0.963 & 1.023 & 1.018 & 1.100 & 0.985 & 0.904 \\
\hline Gen.volt 34 & 0.984 & 1.034 & 1.023 & 0.961 & 1.015 & 1.001 \\
\hline Gen.volt 36 & 0.980 & 1.035 & 1.014 & 1.036 & 1.084 & 1.003 \\
\hline Gen.volt 40 & 0.970 & 1.016 & 1.015 & 1.091 & 0.983 & 0.959 \\
\hline Gen.volt 42 & 0.985 & 1.019 & 1.015 & 0.970 & 1.051 & 1.003 \\
\hline Gen.volt 46 & 1.005 & 1.010 & 1.017 & 1.039 & 0.975 & 1.009 \\
\hline Gen.volt 49 & 1.025 & 1.045 & 1.030 & 1.083 & 0.983 & 1.002 \\
\hline Gen.volt 54 & 0.955 & 1.029 & 1.020 & 0.976 & 0.963 & 0.919 \\
\hline Gen.volt 55 & 0.952 & 1.031 & 1.017 & 1.010 & 0.971 & 0.959 \\
\hline Gen.volt56 & 0.954 & 1.029 & 1.018 & 0.953 & 1.025 & 0.950 \\
\hline Gen.volt 59 & 0.985 & 1.052 & 1.042 & 0.967 & 1.000 & 0.959 \\
\hline Gen.volt 61 & 0.995 & 1.042 & 1.029 & 1.093 & 1.077 & 0.968 \\
\hline Gen.volt 62 & 0.998 & 1.029 & 1.029 & 1.097 & 1.048 & 0.979 \\
\hline
\end{tabular}




\begin{tabular}{|c|c|c|c|c|c|c|}
\hline Gen.volt 65 & 1.005 & 1.054 & 1.042 & 1.089 & 0.968 & 1.002 \\
\hline Gen.volt 66 & 1.050 & 1.056 & 1.054 & 1.086 & 0.964 & 1.000 \\
\hline Gen.volt 69 & 1.035 & 1.072 & 1.058 & 0.966 & 0.957 & 1.031 \\
\hline Gen.volt 70 & 0.984 & 1.040 & 1.031 & 1.078 & 0.976 & 1.030 \\
\hline Gen.volt 72 & 0.980 & 1.039 & 1.039 & 0.950 & 1.024 & 1.031 \\
\hline Gen.volt 73 & 0.991 & 1.028 & 1.015 & 0.972 & 0.965 & 1.027 \\
\hline Gen.volt 74 & 0.958 & 1.032 & 1.029 & 0.971 & 1.073 & 1.029 \\
\hline Gen.volt 76 & 0.943 & 1.005 & 1.021 & 0.960 & 1.030 & 1.027 \\
\hline Gen.volt 77 & 1.006 & 1.038 & 1.026 & 1.078 & 1.027 & 1.029 \\
\hline Gen.volt 80 & 1.040 & 1.049 & 1.038 & 1.078 & 0.985 & 1.032 \\
\hline Gen.volt 85 & 0.985 & 1.024 & 1.024 & 0.956 & 0.983 & 1.021 \\
\hline Gen.volt 87 & 1.015 & 1.019 & 1.022 & 0.964 & 1.088 & 1.023 \\
\hline Gen.volt 89 & 1.000 & 1.074 & 1.061 & 0.974 & 0.989 & 1.039 \\
\hline Gen.volt 90 & 1.005 & 1.045 & 1.032 & 1.024 & 0.990 & 1.037 \\
\hline Gen.volt 91 & 0.980 & 1.052 & 1.033 & 0.961 & 1.028 & 1.004 \\
\hline Gen.volt 92 & 0.990 & 1.058 & 1.038 & 0.956 & 0.976 & 1.031 \\
\hline Gen.volt 99 & 1.010 & 1.023 & 1.037 & 0.954 & 1.088 & 1.029 \\
\hline Gen.volt 100 & 1.017 & 1.049 & 1.037 & 0.958 & 0.961 & 1.033 \\
\hline Gen.volt 103 & 1.010 & 1.045 & 1.031 & 1.016 & 0.961 & 1.029 \\
\hline Gen.volt 104 & 0.971 & 1.035 & 1.031 & 1.099 & 1.012 & 1.004 \\
\hline Gen.volt 105 & 0.965 & 1.043 & 1.029 & 0.969 & 1.068 & 1.039 \\
\hline Gen.volt 107 & 0.952 & 1.023 & 1.008 & 0.965 & 0.976 & 1.032 \\
\hline Gen.volt 110 & 0.973 & 1.032 & 1.028 & 1.087 & 1.041 & 1.014 \\
\hline Gen.volt 111 & 0.980 & 1.035 & 1.039 & 1.037 & 0.979 & 1.011 \\
\hline Gen.volt 112 & 0.975 & 1.018 & 1.019 & 1.092 & 0.976 & 1.092 \\
\hline Gen.volt 113 & 0.993 & 1.043 & 1.027 & 1.075 & 0.972 & 1.004 \\
\hline Gen.volt 116 & 1.005 & 1.011 & 1.031 & 0.959 & 1.033 & 1.004 \\
\hline Tt 8 & 0.985 & 0.999 & 0.994 & 1.011 & 1.004 & 0.929 \\
\hline Tt 32 & 0.960 & 1.017 & 1.013 & 1.090 & 1.060 & 1.003 \\
\hline Tt 36 & 0.960 & 0.994 & 0.997 & 1.003 & 1.000 & 0.939 \\
\hline Tt 51 & 0.935 & 0.998 & 1.000 & 1.000 & 1.000 & 0.940 \\
\hline Tt 93 & 0.960 & 1.000 & 0.997 & 1.008 & 0.992 & 1.012 \\
\hline Tt 95 & 0.985 & 0.995 & 1.020 & 1.032 & 1.007 & 0.963 \\
\hline Tt 102 & 0.935 & 1.024 & 1.004 & 0.944 & 1.061 & 1.024 \\
\hline Tt 107 & 0.935 & 0.989 & 1.008 & 0.906 & 0.930 & 0.926 \\
\hline Tt 127 & 0.935 & 1.010 & 1.009 & 0.967 & 0.957 & 1.015 \\
\hline capacitor reactive power 34 & 0.140 & 0.049 & 0.048 & 0.093 & 0.117 & 0.033 \\
\hline capacitor reactive power 44 & 0.100 & 0.026 & 0.026 & 0.093 & 0.098 & 0.013 \\
\hline capacitor reactive power 45 & 0.100 & 0.196 & 0.197 & 0.086 & 0.094 & 0.159 \\
\hline capacitor reactive power 46 & 0.100 & 0.117 & 0.118 & 0.089 & 0.026 & 0.140 \\
\hline
\end{tabular}




\begin{tabular}{|c|c|c|c|c|c|c|}
\hline capacitor reactive power 48 & 0.150 & 0.056 & 0.056 & 0.118 & 0.028 & 0.038 \\
\hline capacitor reactive power 74 & 0.120 & 0.120 & 0.120 & 0.046 & 0.005 & 0.139 \\
\hline capacitor reactive power 79 & 0.200 & 0.139 & 0.140 & 0.105 & 0.148 & 0.109 \\
\hline capacitor reactive power 82 & 0.200 & 0.180 & 0.180 & 0.164 & 0.194 & 0.149 \\
\hline capacitor reactive power 83 & 0.100 & 0.166 & 0.166 & 0.096 & 0.069 & 0.139 \\
\hline capacitor reactive power 105 & 0.200 & 0.189 & 0.190 & 0.089 & 0.090 & 0.143 \\
\hline capacitor reactive power 107 & 0.060 & 0.128 & 0.129 & 0.050 & 0.049 & 0.133 \\
\hline capacitor reactive power 110 & 0.060 & 0.014 & 0.014 & 0.055 & 0.022 & 0.010 \\
\hline PG (MW) & 4374.8 & 4359.3 & 4361.4 & $\mathrm{NR}^{*}$ & $\mathrm{NR}^{*}$ & 4381.12 \\
\hline QG (MVAR) & 795.6 & 604.3 & 653.5 & $* \mathrm{NR}^{*}$ & $\mathrm{NR}^{*}$ & 612.23 \\
\hline Reduction in PLOSS (\%) & 0 & 11.7 & 10.1 & 0.6 & 1.3 & 14.55 \\
\hline Total PLOSS (Mw) & 132.8 & 117.19 & 119.34 & 131.99 & 130.96 & 113.47 \\
\hline
\end{tabular}

NR* - Not reported.

Then IEEE 300 bus system is used as test system to validate the performance of the Hybridization of Tree-seed algorithm with sine-cosine algorithm (HTS) has been tested without considering voltage stability index. Table 11 shows the comparison of real power loss obtained after optimization.

Table 11. Comparison of Real Power Loss

\begin{tabular}{lllll}
\hline & EGA [58] & EEA [58] & CSA [57] & HTS \\
\hline PLOSS (MW) & 646.2998 & 650.6027 & 635.8942 & 611.7902 \\
\hline
\end{tabular}

\section{Conclusion and future scope of work}

Real power Loss has been achieved by proposed Hybridization of Tree-seed algorithm with sine-cosine algorithm (HTS). In Tree-seed algorithm, a converse correlation has been maintained between the exploration and exploitation. In search of the optimal values of the dispersion of seeds, it is very fundamental and number of seeds updating will start from maximum and it will be regularly reduced. Then in this work, a new linear parameter " $\mathrm{K}$ " has been implemented and it inspired from sine cosine algorithm which induces the solution to jump out from local environment. At first in the procedure, exploration has been improved then gradually exploitation will be enhanced. In proposed work throughout the iterative procedure both the exploration and exploitation has been balanced. In IEEE 30, bus system proposed HTS algorithm has been evaluated. Then the Proposed Hybridization of Tree-seed algorithm with sine-cosine algorithm (HTS) has been tested in standard IEEE 14, 30, 57, 118, 300 bus test systems without considering the voltage stability index. Power loss reduction achieved along with voltage stability enhancement. Mainly voltage deviation minimization also attained. In future, this work can be extended by applying the hybrid algorithm to solve the problem in real time mode. Practical systems can be analyzed and evaluated by the proposed approach.

\section{References}

[1] K. Y. Lee. (1984). "Fuel-cost minimisation for both real and reactive-power dispatches,” Proceedings Generation, Transmission and Distribution Conference, 131(3), pp. 85-93.

[2] N. I. Deeb. (1998). “An efficient technique for reactive power dispatch using a revised linear programming approach,” Electric Power System Research, 15(2), pp. 121-134.

[3] M. R. Bjelogrlic, M. S. Calovic, B. S. Babic. (1990). “Application of Newton’s optimal power flow in voltage/reactive power control”, IEEE Trans Power System, vol. 5, no. 4, pp. 1447-1454.

[4] S. Granville. (1994). “Optimal reactive dispatch through interior point methods,” IEEE Transactions on Power System, 9(1), pp. 136-146. http://dx.doi.org/10.1109/59.317548.

[5] N. Grudinin. (1998). "Reactive power optimization using successive quadratic programming method," IEEE Transactions on Power System, 13(4), pp. 1219-1225. http://dx.doi.org/10.1109/59.736232. 
[6] Uğurarifoğlu and farukyalçin. (2018). System Constrained Active Power Loss Minimization in Practical Multi-terminal HVDC Systems through GA, Sakarya University Journal of Science, 10.16984/saufenbilder.421351, (1-1), (2018).

[7] Kamel, S., Abdel-Fatah, S., Ebeed, M., Yu, J., Xie, K. G., and Zhao, C. Y. (2019). Solving Optimal Reactive Power Dispatch Problem Considering Load Uncertainty. 10.1109/ISGT-Asia.2019.8881322.

[8] Biplab Bhattacharyya and Nihar Karmakar. (2019). Optimal Reactive Power Management Problem: A Solution Using Evolutionary Algorithms, IETE Technical Review. DOI: 10.1080/02564602.2019.1675541.

[9] S. Kamel, S. Abdel-Fatah, M. Ebeed, J. Yu, K. Xie, and C. Zhao. (2019). “Solving Optimal Reactive Power Dispatch Problem Considering Load Uncertainty,” 2019 IEEE Innovative Smart Grid Technologies - Asia (ISGT Asia), Chengdu, China, 2019, pp. 1335-1340, doi: 10.1109/ISGT-Asia.2019.8881322.

[10] T. M. Aljohani, A. F. Ebrahim, O. Mohammed Single. (2019). "Multiobjective Optimal Reactive Power Dispatch Based on Hybrid Artificial Physics-Particle Swarm Optimization”, Energies, 12(12), 2333; https://doi.org/10.3390/en12122333.

[11] Ram Kishan Mahate and Himmat Singh. (2019). Multi-Objective Optimal Reactive Power Dispatch Using Differential Evolution. International Journal of Engineering Technologies and Management Research, 6(2), 27-38. http://doi.org/10.5281/zenodo.2585477.

[12] Nguyen, ThangTrung, Vo, Dieu Ngoc. (n.d.). "Improved social spider optimization algorithm for optimal reactive power dispatch problem with different objectives”. Neural Computing and Applications, VL-32, IS-10. https://doi.org/10.1007/s00521-019-04073-4.

[13] Yang, S., Wang, W., Liu, C., et al. Optimal reactive power dispatch of wind power plant cluster considering static voltage stability for low-carbon power system. J. Mod. Power Syst. Clean Energy3, 114-122 (2015). https://doi.org/10.1007/s40565-014-0091-x

[14] S. Emiroglu, Y. Uyaroglu, G. Ozdemir. (2017). "Distributed Reactive Power Control based Conservation Voltage Reduction in Active Distribution Systems,” Advances in Electrical and Computer Engineering, vol. 17, no. 4, pp. 99-106. doi:10.4316/AECE.2017.04012.

[15] Mojtaba Ghasemi, Mahdi Taghizadeh, Sahand Ghavidel, Jamshid Aghaei, Abbas Abbasian. (2015). "Solving optimal reactive power dispatch problem using a novel teaching-learning-based optimization algorithm”, Engineering Applications of Artificial Intelligence, Volume 39, pp. 100-108.

[16] Wei, Yan-Ling, Nguyen, Thang Trung, Vo, Dieu Ngoc, Van Tran, Hai, Van Dai, Le. (2019). “Optimal Dispatch of Reactive Power Using Modified Stochastic Fractal Search Algorithm”, Complexity, Hindaw, 1076-2787, 2019.

[17] A. Padilha-Feltrin, D. A. QuijanoRodezno, and J. R. S. Mantovani. (2015). "Volt-VAR Multiobjective Optimization to Peak-Load Relief and Energy Efficiency in Distribution Networks,” IEEE Transactions on Power Delivery, vol. 30, no. 2, pp. 618-626, Apr. 2015.

[18] Ghazavi Dozein, M., Monsef, H., Ansari, J., and Kazemi, A. (2016). An effective decentralized scheme to monitor and control the reactive power flow: a holonic-based strategy. Int. Trans. Electr. Energ. Syst., 26: 1184-1209.

[19] S. D. Beigvand, H. Abdi, and M. La Scala. (2016). “Combined heat and power economic dispatch problem using gravitational search algorithm,” Electr. Power Syst. Res., 133, 160-172.

[20] N. Narang, E. Sharma, and J. S. Dhillon. (2017). “Combined heat and power economic dispatch using integrated civilized swarm optimization and Powell’s pattern search method,” Appl. Soft Comput., 52, 190-202.

[21] W. Warid, H. Hizam, N. Mariun, and N. I. A. Wahab. (2018). “A novel quasi-oppositional modified Jaya algorithm for multi-objective optimal power flow solution,” Applied Soft Computing Journal, Vol. 65, pp. 360-373, Apr. 2018.

[22] O. Herbadji, L. Slimani, and T. Bouktir. (2017). "Multiobjective optimal power flow considering the fuel cost, emission, voltage deviation and power losses using multi-objective dragonfly algorithm”, International Conference on Recent Advances in Electrical Systems, pp. 191-197, 2017.

[23] K. Vaisakh, Member, IEEE, and P. Kanta Rao. (n.d.). “Optimum Reactive Power Dispatch Using Differential Evolution for Improvement of Voltage Stability”, 978-1-4244-1762-9/08/ C 2008 IEEE.

[24] S. D. Chavan, Nisha P. Adgokar. (2015). “An Overview on Particle Swarm Optimization: Basic Concepts and Modified Variants”. International Journal of Science and Research, Volume 4 Issue 5, May 2015, pp. 255-260.

[25] Nagendra, P., Halder Nee Dey, S., and Paul, S. (2014). "Voltage stability assessment of a power system incorporating FACTS controllers using unique network equivalent”, Ain Shams Eng. J., 5(1), 103-111.

[26] Nagendra, P., Halder Nee Dey, S., and Paul, S. (2015). "Location of static VAR compensator in a multi-bus power system using unique network equivalent”, Adv. Energy Res., 3(4), 235-249.

[27] H. Zhang, X. Lei, C. Wang, D. Yue, and X. Xie. (2017). “Adaptive grid based multi-objective Cauchy differential evolution for stochastic dynamic economic emission dispatch with wind power uncertainty,” PLOS ONE, pp. 1-25, 29 September 2017.

[28] K. N. Bindu and K. K. Kumar. (2016). “Combined Economic And Emission Dispatch Using Random Drift Particle Swarm 
Optimization,” International Journal for Modern Trends in Science and Technology, vol. 2, no. 11, pp. 134-139, November 2016.

[29] Rupa, J. M., Ganesh, S. (2014). Power flow analysis for radial distribution system using backward/forward sweep method. Inter J Electr, Comput, Electron Commun Eng., 8: 1540-1544.

[30] Abdel-Akher, M. (2013). Voltage stability analysis of unbalanced distribution systems using backward/forward sweep load-flow analysis method with secant predictor. IET gener, transmdistrib., 7: 309-317.

[31] Prasad, B., C. D. Prasad, and G. P. Kumar. (2016). "Effect of load parameters variations on AGC of single area thermal power system in presence of integral and PSO-PID controllers,” 2015 Conf. Power, Control. Common. Compute. Technol. Sustain. Growth, PCCCTSG 2015, no. 1, pp. 64-68, 2016.

[32] Javadmorsal, Kazemzare, Mehrdad Tarafdar Hagh. (2016). "Performance comparison of TCSC with TCPS and SSSC controllers in AGC of realistic interconnected multi-sources power system,” Elsevier, pp. 64-68, 2016.

[33] Uğurarifoğlu and Farukyalçin. (2018). System Constrained Active Power Loss Minimization in Practical Multi-terminal HVDC Systems through GA, Sakarya University Journal of Science, 10.16984/saufenbilder.421351, (1-1), (2018).

[34] Wei, H., Lin, C., and Wang, Y. (2018). The optimal reactive power flow model in mixed polar form based on transformer dummy nodes. IEEJ Trans Elec Electron Eng., 13: 411-416.

[35] Fang, S., Cheng, H., Xu, G., Zhou, Q., He, H., Zeng, P. (2017). Stochastic optimal reactive power reserve dispatch considering voltage control areas. Int. Trans. Electr. Energ. Syst., 2017; 27: e2269.

[36] Ghazavi Dozein, M., Monsef, H., Ansari, J., and Kazemi, A. (2016). An effective decentralized scheme to monitor and control the reactive power flow: a holonic-based strategy. Int. Trans. Electr. Energ. Syst., 26: 1184-1209.

[37] Du, Z., Nie, Y., and Liao, P. (2014). PCPDIPM-based optimal reactive power flow model using augmented rectangular coordinates. Int. Trans. Electr. Energ. Syst., 24: 597-608.

[38] Liu, B., Liu, F., Zhai, B., and Lan, H. (2019). Investigating continuous power flow solutions of IEEE 16us system. IEEJ Trans Elec Electron Eng., 14: 157-159.

[39] A. Panda, S. Pani. (2016). A symbiotic organisms search algorithm with adaptive penalty function to solve multi-objective constrained optimization problems, Appl. Soft Comput., 46(2016): 344-360.

[40] C. K. Shiva, V. Mukherjee. (2015). A novel quasi-oppositional harmony search algorithm for automatic generation control of power system, Appl. Soft Comput., 35(2015): 749-765.

[41] H. R. E. H. Bouchekara, M. Zellagui, and M. A. Abido. (2017). "Optimal coordination of directional overcurrent relays using a modified electromagnetic field optimization algorithm,” Applied Soft Computing, vol. 54, pp. 267-283, 2017.

[42] B. Talebi and M. N. Dehkordi. (2018). "Sensitive association rules hiding using electromagnetic field optimization algorithm," Expert Systems with Applications, vol. 114, pp. 155-172, 2018.

[43] Becerra, V. Cooray. (2006). “A simplified physical model to determine the lightning upward connecting leader inception”, IEEE Trans. Power Deliv., 21, 897-908.

[44] M. Kiran. (2015). “TSA: tree-seed algorithm for continuous optimization”, Expert Syst. Appl., 42(19), 6686-6698.

[45] A. Babalik, A. Cinar, M. Kiran. (2018). A modification of tree-seed algorithm using Deb’s rules for constrained optimization. Appl. Soft Comput., 63(2018), 289-305.

[46] M. Aslan, M. Beskirli, H. Kodaz, M. Kiran. (2018). An improved tree seed algorithm for optimization problems, Int. J. Mach. Learn. Comput., 8(1): 20-25.

[47] Jiang, J. H., Xu, M. R., Meng, Xian. Q., and Li, K. Q. (2019). STSA: A sine Tree-Seed Algorithm for complex continuous optimization problems. Physica A: Statistical Mechanics and its Applications. 537. 122802. 10.1016/j.physa.2019.122802.

[48] S. Mirjalili. (2016). SCA: a sine cosine algorithm for solving optimization problems. Knowledge-Based System, 96(2016), 120-133.

[49] Illinois Center for a Smarter Electric Grid (ICSEG). Available online: https://icseg.iti.illinois.edu/ieee-30-bussystem/ (accessed on 25 February 2019).

[50] El Ela, A. A., Abido, M. A., Spea, S. R. (2011). Differential evolution algorithm for optimal reactive power dispatch. Electr. Power Syst. Res., 2011, 81, 458-464.

[51] Duman, S., Sönmez, Y., Güvenç, U., Yörükeren, N. (2012). Optimal reactive power dispatch using a gravitational search algorithm. IET Gener. Transm. Distrib., 2012, 6, 563-576.

[52] Aljohani, T. M., Ebrahim, A. F., Mohammed, O. (2019). Single and Multiobjective Optimal Reactive Power Dispatch Based on Hybrid Artificial Physics-Particle Swarm Optimization. Energies, 2019, 12, 2333.

[53] Dai, C., W. Chen, Y. Zhu, and X. Zhang. (2009). Seeker optimization algorithm for optimal reactive power dispatch. IEEE T. 
Power Syst., 24(3): 1218-1231.

[54] Subbaraj, P. and P. N. Rajnarayan. (2009). Optimal reactive power dispatch using self-adaptive real coded Genetic algorithm. Electr. Power Syst. Res., 79(2): 374-38.

[55] Pandya, S. and R. Roy. (2015). Particle swarm optimization based optimal reactive power dispatch. Proceeding of the IEEE International Conference on Electrical, Computer and Communication Technologies (ICECCT), pp. 1-5.

[56] Ali Nasser Hussain, Ali Abdulabbas Abdullah, and Omar Muhammed Neda. (2018). "Modified Particle Swarm Optimization for Solution of Reactive Power Dispatch”, Research Journal of Applied Sciences, Engineering and Technology 15(8): 316-327, 2018. DOI:10.19026/rjaset.15.5917.

[57] S. Surender Reddy. (2017). “Optimal Reactive Power Scheduling Using Cuckoo Search Algorithm”, International Journal of Electrical and Computer Engineering, Vol. 7, No. 5, pp. 2349-2356.

[58] S. S. Reddy. (2014). "Faster evolutionary algorithm based optimal power flow using incremental variables”, Electrical Power and Energy Systems, vol. 54, pp. 198-210. 